\title{
The effect of motion on tactile and visual temporal order judgments
}

\author{
JAMES C. CRAIG and THOMAS A. BUSEY \\ Indiana University, Bloomington, Indiana
}

\begin{abstract}
Four experiments were conducted, three with tactile stimuli and one with visual stimuli, in which subjects made temporal order judgments (TOJs). The tactile stimuli were patterns that moved laterally across the fingerpads. The subject's task was to judge which finger received the pattern first. Even though the movement was irrelevant to the task, the subjects' TOJs were greatly affected by the direction of movement of the patterns. Accuracy in judging temporal order was enhanced when the patterns moved in a direction that was consistent with the temporal order of presentation-for example, when the movement on each fingerpad was from right to left and the temporally leading site of stimulation was to the right of the temporally trailing site of stimulation. When movement was inconsistent with the temporal order of presentation, accuracy was considerably reduced, often well below chance. The bias in TOJs was unaffected by training or by presenting the stimuli to fingers on opposite hands. In a fourth experiment, subjects judged the temporal order of visual stimuli that, like the tactile stimuli, moved in a direction that was either consistent or inconsistent with the TOJ. The results were similar to those obtained with tactile stimuli. It is suggested that the bias may be affected by attentional mechanisms and by apparent motion generated between the two sites on the skin.
\end{abstract}

Studies of temporal order for tactile stimuli have examined subjects' accuracy in reporting which one of two sites on the skin received a stimulus first (Hill, 1971; Hill \& Bliss, 1968; Hirsh \& Sherrick, 1961; Marks et al., 1982). In these studies, the tactile stimuli were generally a tap or a brief electrical stimulus. In all of the previous tactile studies of which we are aware, the stimuli were presented statically - that is, the stimulus was delivered to a particular site and remained at that site until it was turned off. However, most of the information that is derived from the sense of touch comes by means of patterns moving across the surface of the skin. During haptic exploration, tactile stimuli move sequentially across the skin in what is referred to as a scanned mode (Craig, 2002).

Previous studies of tactile pattern identification have examined a number of modes of generating patterns and, in particular, two modes: the scanned mode and the static mode. The static mode is one in which all the elements defining the pattern are generated at the same location on the skin and remain on at that location (Craig, 1980, 2002). A recent study compared these two modes. The study reported that such perceptual mechanisms as temporal integration, temporal masking, feature displacement, and response competition affected pattern

This research was supported by National Institutes of Health Grant DC00095, National Institute on Deafness and Other Communication Disorders. We thank Roger Rhodes for his assistance in these experiments. Correspondence concerning this article should be addressed to J. C. Craig, Department of Psychology, Indiana University, Bloomington, IN 47405 (e-mail: craigj@indiana.edu). perception to similar degrees, whether the patterns were generated in the scanned or the static mode (Craig, 2002). With the types of patterns generated, there seemed to be no advantage to presenting patterns in the scanned mode, as compared with the static mode; however, with regard to the task of judging temporal order, there is a close relationship between the direction in which patterns are scanned across the skin's surface and the order in which patterns arrive at the skin. Imagine that one is moving the index and middle fingers of the left hand rapidly over an object. If the hand is moving to the left, the surface features of the object will be scanned across the fingertips from left to right, and these features will generally be encountered by the middle finger before being encountered by the index finger. The temporal order-which location (finger) was stimulated first-is completely correlated with the direction of movement. If the hand were moving in the opposite direction - that is, moving to the rightthe left index finger would encounter surface features prior to the middle finger. In general, in haptic exploration, subjects can judge which finger received a stimulus first by simply noting the direction in which the hand was moving and/or the consequent direction of motion across the skin surface. This same relationship holds true for stimuli as they are scanned visually.

The present study was undertaken to examine what role, if any, scanned movement plays in temporal order judgments (TOJs). In these experiments, the subject's task was to judge which of two locations received a stimulus first-a traditional TOJ (Hirsh \& Sherrick, 1961). For most of the experiments, scanned patterns were used. With scanned patterns, the direction of movement could 
be either consistent with the temporal order of the patterns or inconsistent. By consistent is meant that if the first pattern is presented to the left middle finger and the second pattern to the left index finger, the pattern of motion generated on the fingers is from left to right. This is represented in Figure 1A. It is as though the left hand were moving to the left and, thus, the middle finger encounters a feature before the index finger does. For inconsistent movement, the pattern of movement would be in the reverse direction, from right to left; however, the middle finger would still receive the pattern first (Figure 1C). The correct TOJ in both cases would be middle finger first. The question is, would the direction of movement (which is, in fact, irrelevant to the TOJ) affect subjects' judgments?

As was noted, if the hand is moved across a surface, temporal order and direction of movement are often highly correlated. To generate movement across the skin without hand movements, stimuli were generated on two tactile arrays contacting the two fingers. The subject's task was always to report which one of the two sites (fingers) was stimulated first. The direction of motion was irrelevant to the TOJ.

One reason to expect the direction of movement to affect TOJs is, as was noted, the tight coupling between the hand or object movements and which sites on the skin receive a stimulus first. Also, pattern movement might affect TOJs because apparent motion often accompanies TOJs. The conditions under which temporal order is studied, the presentation of stimuli to two sites on the skin with a temporal separation between them, are the conditions that can generate apparent motion (Sherrick, 1968a, 1968b). Subjects in temporal order studies often report apparent motion (Craig \& Xu, 1990), and such motion is often used as a cue to judging temporal order (Sherrick, 1970).

\section{EXPERIMENT 1}

In the course of haptic exploration, adjacent fingers on the same hand will contact the same feature at slightly different times, and there is a high likelihood that the same feature will contact both fingers. If movement cues associated with haptic exploration affect TOJs, this effect should be most obvious when the sites of stimulation are adjacent fingers on the same hand. These were the sites used in Experiment 1.

Subjects were asked to judge which one of two fingers received a pattern first. The patterns at each finger moved laterally across the fingerpad. On some trials, the temporal order was consistent with the movement of the patterns across the two fingers that would be generated by haptic exploration. On other trials, the temporal order was inconsistent with movement and haptic exploration.

Three conditions were tested. In the first condition, the subjects made TOJs without trial-by-trial feedback. In the second condition, the subjects received trial-bytrial feedback. In the first and second conditions, the

\section{Consistent Movement}

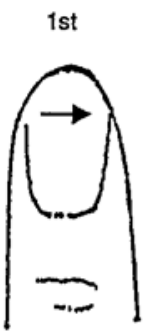

A

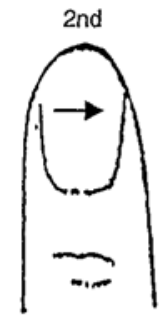

\section{Inconsistent Movement}
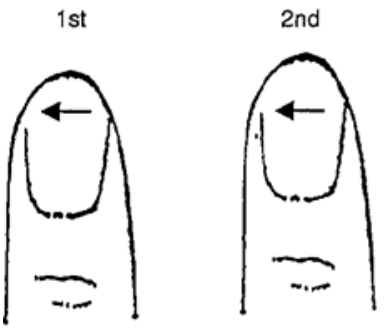

C

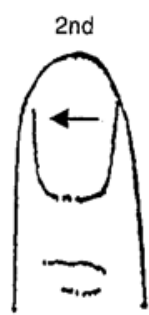

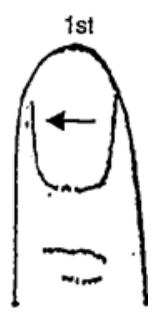

$B$

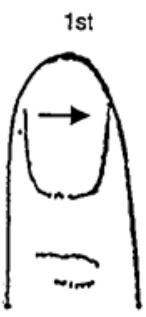

Figure 1. Representations of the directions of movement across the middle and index fingers of the left hand. Panels $A$ and $B$ represent conditions in which the direction of movement is consistent with the temporal order of presentation of the stimuli. The temporal order of presentation of the stimuli is marked " $1 \mathrm{st}$ " and "2nd." Panels C and D represent conditions in which the direction of movement is inconsistent with the temporal order. 
movement at the two sites was always in the same direction, movement at both sites being either to the left or to the right. In a third condition, the movements at the two sites were in opposite directions to one another, with the patterns at the two fingers moving either toward one another or away from one another. In this condition, the temporally leading pattern could be consistent with the temporal order and the trailing pattern inconsistent, or the leading pattern could be inconsistent with the temporal order and the trailing pattern consistent. The results from the third condition were analyzed to see whether patterns moving toward one another would bias TOJs and to see whether the leading pattern exerted more of an influence on the TOJs than did the trailing pattern.

\section{Method}

Subjects. The subjects were undergraduate students at Indiana University. They were paid an hourly wage for their participation. The subjects were generally experienced in tactile pattern recognition tasks and in judging temporal order.

Apparatus. The tactile stimuli were generated on two tactile displays interfaced with a PC. Each display consisted of 144 tactors arranged in a 6-column $\times 24$-row array. The displays were similar to those used in Optacons, reading aids for the blind (Bliss, Katcher, Rogers, \& Shepard, 1970). The arrays fit against the distal portion of the subjects' fingerpads. Only the top 15 rows of the displays were used, and these covered an area of $17 \times 11 \mathrm{~mm}$. When activated, each tactor vibrated at 230 pulses per second.

Procedure. The subject's task was to indicate which one of the two fingers received the stimulus first. The subjects rested the index and middle fingers of their left hands on the tactile arrays. Moving patterns were generated by activating columns sequentially on the Optacon display. A representation of the type of pattern used is shown in Figure 2. Each pair of columns was activated for $13 \mathrm{msec}$ with a stimulus onset asynchrony (SOA) of $13 \mathrm{msec}$ between columns. The total pattern duration was $65 \mathrm{msec}$. The sensation was one of a light mechanical stimulus moving across the skin (Gardner \& Sklar, 1994). Following each presentation of the pair of stimuli, the subjects responded by pressing " 1 " or " 2 " on a keyboard, corresponding to the middle and the index fingers, respectively. The subjects were told that the direction of movement was irrelevant to the TOJ.

Three conditions were tested. In the first two conditions, the patterns presented to the two fingers moved in the same direction on both fingers, from left to right or from right to left. The direction of motion was uncorrelated with the order of presentation at the two fingers - that is, temporal order. The subjects received no feedback in this condition. The second condition was identical to the first with the addition of trial-by-trial feedback. The feedback consisted of the word correct or, if incorrect, the number of the finger- 1 for the middle finger, 2 for the index finger - that was the site of the leading stimulus. In a third condition, the patterns moved in opposite directions from each other. On approximately half of the trials, the two patterns moved toward each other, and on the other half of the trials, the patterns moved away from each other.

Five SOAs were tested: 200, 100, 52, 26, and $13 \mathrm{msec}$. Trials were blocked by SOA, with 50 trials per block. Each experimental session tested the five SOAs in order from longest to shortest. We began with the longest SOA, an SOA at which the subjects were generally correct, in order to help the subjects get a clear picture of the task and to underscore the fact that the directions of motion on the fingerpads were irrelevant to the TOJ. The same subjects were tested in all three conditions. Table 1 shows the number of subjects, sessions, and the number of trials per SOA for each of the three conditions.

\section{Results and Discussion}

The results from the first condition are shown in Figure 3. The percentage of correct responses is plotted as a function of the SOA. Three functions are shown: the percentage correct on those trials in which the movement of the patterns was consistent with the TOJ, the percentage of trials on which the movement was inconsistent with the TOJ, and the mean of those two functions. The latter function is approximately equal to the overall percentage of correct responses. It is clear that the direction of the movement at the two fingerpads has a large effect on TOJs. An analysis of variance showed a significant effect of SOA $[F(4,24)=41.89, p<.001]$, a significant effect of trial type [consistent vs. inconsistent; $F(1,6)=75.24$, $p<.001]$, and a significant interaction between trial type and SOA $[F(4,24)=16.21, p<.001]$. The interaction ap-

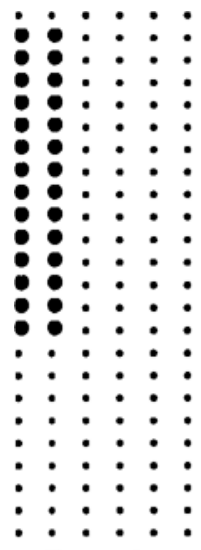

Frame 1

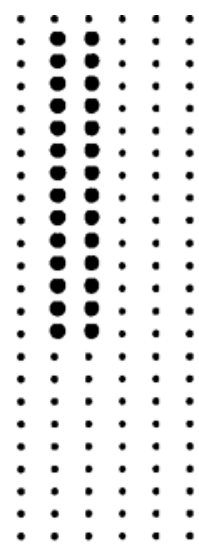

Frame 2

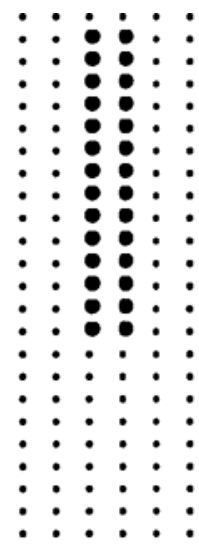

Frame 3

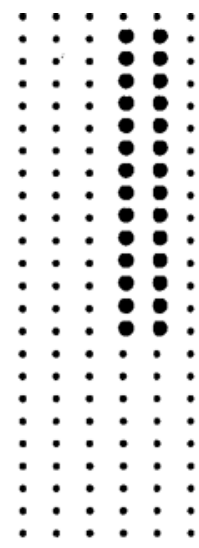

Frame 4

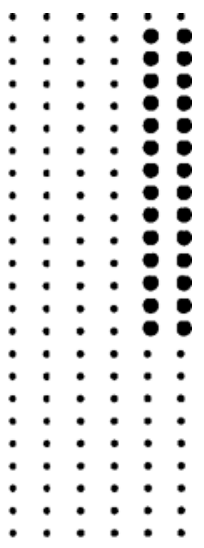

Frame 5

Figure 2. A representation of the moving patterns. Each panel represents one frame of the tactile stimulus. 
Table 1

Experimental Conditions

\begin{tabular}{ccccccr}
\hline Experiment & Condition & Ipsi-/Bilateral & Scan/Static & Subjects & Sessions & \multicolumn{1}{c}{ Trials } \\
\hline 1 & 1 & ipsilateral & scan & $5 \mathrm{~F}, 2 \mathrm{M}$ & 6 & $1,050 / \mathrm{SOA}$ \\
1 & 2 & ipsilateral & scan & $5 \mathrm{~F}, 2 \mathrm{M}$ & 4 & $700 / \mathrm{SOA}$ \\
1 & 3 & ipsilateral & scan/opposed & $5 \mathrm{~F}, 2 \mathrm{M}$ & 4 & $700 / \mathrm{SOA}$ \\
2 & 1 & ipsilateral & static & $5 \mathrm{~F}, 3 \mathrm{M}$ & 5 & $2,000 / \mathrm{SOA}$ \\
2 & 2 & ipsilateral & scan & $5 \mathrm{~F}, 3 \mathrm{M}$ & 5 & $1,000 / \mathrm{SOA}$ \\
2 & 3 & ipsilateral & scan/static & $5 \mathrm{~F}, 3 \mathrm{M}$ & 7 & $700 / \mathrm{SOA}$ \\
3 & 1 & bilateral/28 cm & scan & $4 \mathrm{~F}, 2 \mathrm{M}$ & 4 & $700 / \mathrm{SOA}$ \\
3 & 2 & bilateral/28 cm & scan & $6 \mathrm{~F}, 2 \mathrm{M}$ & 4 & $700 / \mathrm{SOA}$ \\
3 & 3 & bilateral/80 cm & scan & $4 \mathrm{~F}, 2 \mathrm{M}$ & 5 & $875 / \mathrm{SOA}$ \\
4 & 1 & visual & scan & $9 \mathrm{~F}, 3 \mathrm{M}$ & 5 & $960 / \mathrm{SOA}$ \\
\hline
\end{tabular}

pears to be the result of the improved performance at the longer SOAs on inconsistent trials. The effect of movement is most clearly seen at the briefer SOAs, where the TOJs are uncertain. It appears that the TOJs are a joint function of the temporal order of the two stimuli and a weighting factor resulting from the direction of motion. The weighting factor declines with increasing SOA.

What these results make clear is that subjects make use of movement in judging which one of two locations received a stimulus first. Indeed, at brief SOAs, they rely almost entirely on the direction of movement. In the normal course of haptic exploration, the direction of movement on the fingers on one hand is nearly perfectly correlated with the temporal order.

Another way to look at these results is to ask how much of a temporal separation is required on inconsistent trials to achieve the same percentage of correct responses as that obtained on consistent trials. Usually, one would compare performance at the $75 \%$ point, halfway between chance and $100 \%$ correct; this would be analogous to calculating the point of subjective equality. However, for the results shown in Figure 3, the consistent movement function does not drop below $82 \%$ correct. For the consistent function, an SOA of $13 \mathrm{msec}$ produced $82 \%$ correct, whereas the inconsistent function did not reach $82 \%$ correct until an SOA estimated to be $187 \mathrm{msec}$. In other words, to overcome the bias produced by the direction of motion required an increase in the time that the first stimulus led the second from 13 to $187 \mathrm{msec}$, or $174 \mathrm{msec}$. We also analyzed the data to see whether there was a bias to respond with one of the two locations more than with the other. Overall, the subjects responded that the middle finger was first on $52 \%$ of the trials and that the index finger was first on the remaining trials, $48 \%$.

The results from the second condition, in which trialby-trial feedback was provided, are shown in Figure 4. The results are plotted in the same way as in Figure 3, and similarly, there is a large effect of movement on TOJs.

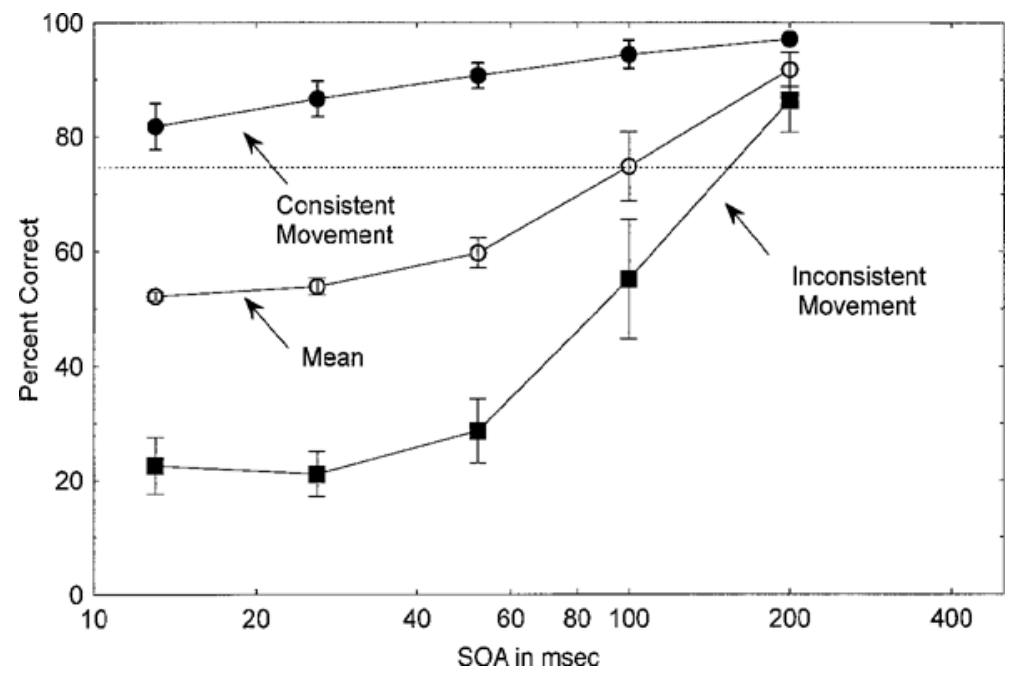

Figure 3. Percentages correct in judging temporal order as a function of the stimulus onset asynchrony (SOA) in milliseconds. The top function represents those trials on which the direction of movement of the stimuli was consistent with the order of presentation of the stimuli. The bottom function represents those trials on which the direction of movement was inconsistent with the order of presentation. The middle function represents the mean of the consistent and inconsistent functions. Error bars represent \pm 1 standard error of the mean. 


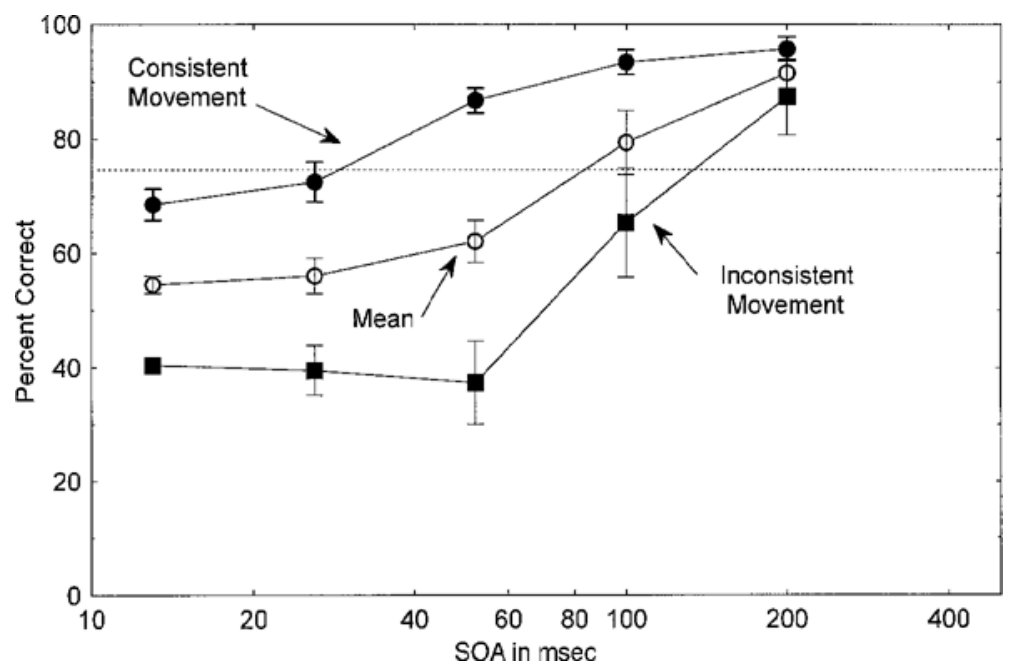

Figure 4. Performance in judging temporal order with trial-by-trial feedback. The top function represents consistent trials, the bottom function represents inconsistent trials, and the middle function represents the mean of the consistent and inconsistent trials. Error bars represent \pm 1 standard error of the mean.

An analysis of variance showed a pattern of results similar to those obtained with feedback: a main effect of SOA $[F(4,24)=29.56, p<.001]$, a main effect of trial type $[F(1,6)=49.77, p<.001]$, and a significant interaction between SOA and trial type $[F(4,24)=8.46, p<.001]$. Even with trial-by-trial feedback, the subjects' responses were greatly affected by the direction of motion of the stimuli whose temporal order they were judging. It should be noted that these were experienced subjects in that they had had considerable experience in making TOJs, yet their performance continued to be affected by movement at the two sites of stimulation, movement that was irrelevant to the task.

The results from the third condition are shown in Figure 5. Two functions are shown. The leading stimulus consistent function represents performance on those trials on which the temporally leading stimulus was moving in the direction consistent with the TOJ-for example, movement on the index finger to the left (toward the middle finger) when the index finger received the pat-

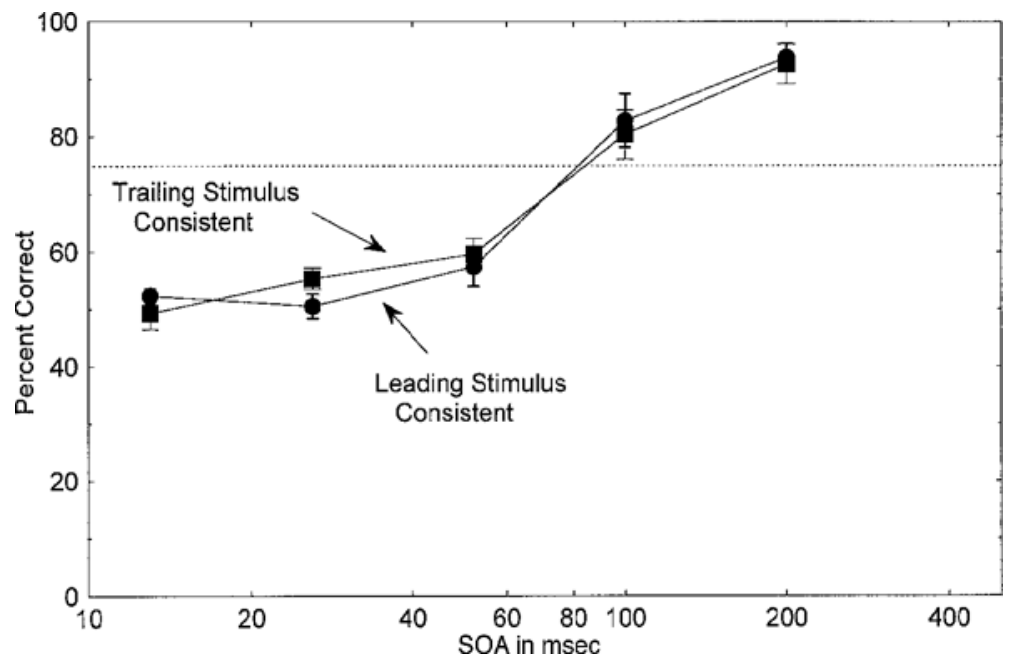

Figure 5. Performance in judging temporal order. The stimulus patterns were moving in opposite directions. For the function marked "leading stimulus consistent," the leading stimulus was moving in a direction consistent with the temporal order of the two stimuli. For the function marked "trailing stimulus consistent," the trailing stimulus was moving in a direction consistent with the temporal order of the two stimuli. Error bars represent \pm 1 standard error of the mean. 
tern first. On those trials, the trailing stimulus is generating movement in the opposite direction (inconsistent movement). The trailing stimulus consistent function represents those trials on which the temporally trailing stimulus is moving in a direction consistent with the TOJ. On those trials, the trailing stimulus would be providing consistent movement cues. As Figure 5 shows with the two patterns moving in opposite directions, there was no differential effect of movement on TOJs, and there was no overall effect of having the leading stimulus consistent or the trailing stimulus consistent $[F(1,6)=0.007, p=.94]$. In other words, there is no indication that the leading pattern had a greater effect on TOJs than did the trailing pattern and vice versa. The results are very similar to the mean results presented in Figures 3 and 4. It appears as though the movements at the two sites canceled one another. These results also indicate that movement was being processed at both sites and affected the TOJs, although as will be seen in Experiment 2 , movement at a single site is sufficient to produce the effect.

From the overall percentage correct functions shown in Figures 3, 4, and 5, the 75\% correct points were calculated by linear interpolation. For Conditions 1, 2, and 3 , these are 101,88 , and $89 \mathrm{msec}$, respectively. The focus of the present study was on the effect that movement has in biasing TOJs, rather than on the overall sensitivity of subjects in making TOJs. Some studies have reported thresholds as low as 20-30 msec (Craig \& Xu, 1990; Hirsh \& Sherrick, 1961). There are a number of variables that affect the threshold for temporal order, and some of the reasons for what appears to be the relatively poor performance with these moving stimuli were addressed in Experiment 2.

\section{EXPERIMENT 2}

Experiment 2 addressed several issues raised in Experiment 1 . The first issue was the overall level of performance achieved in Experiment 1. As was noted, the thresholds with these moving patterns were higher than those that other studies have reported. The effect of movement on TOJs was greatest at the briefer SOAs, where overall performance was relatively poor. It is possible that, with improved performance, the effect of movement might disappear. Initially, it was thought that, in addition to procedural differences, the difficulty might be with the scanned patterns themselves. TOJs of moving patterns might be more inaccurate because of increased uncertainty in judging the onsets of the patterns. It might be simply more difficult to judge the temporal order of moving patterns, as compared with static patterns. We conducted some preliminary measurements with static patterns. We tested static patterns presented for the same duration as the moving patterns and static patterns presented for briefer durations. Neither of these two manipulations produced substantial improvements in performance. An earlier study noted that the training of subjects resulted in significant improvement in TOJs. The training in this previous study consisted of discussing various cues subjects might use in making their judgments and discussing how these cues might change with SOA (Craig \& Xu, 1990). In Experiment 2, we tested a group of subjects with static patterns (Condition 1 ) and then with scanned patterns (Condition 2). With both sets of patterns, we encouraged subjects to discuss with the experimenters the various cues that they were using. We offered suggestions to the subjects for improving their performance, including those suggestions offered by the other subjects.

The second issue we addressed was whether movement was necessary at both sites - that is, could the bias be elicited by movement at just one site? In this condition, the subjects were tested as they had been in Experiment 1, but at only one of the two sites was a moving stimulus presented. The second site received a static stimulusthat is, a stimulus that did not move laterally across the fingerpad. On some trials within a block, the moving pattern was presented first, and on other trials, the moving pattern was presented second. This condition also allowed us to compare directly trials on which the leading stimulus was either consistent or inconsistent with temporal order with trials on which the trailing stimulus was either consistent or inconsistent with temporal order. With this comparison, one could see whether the leading or the trailing moving pattern was more effective in biasing TOJs.

\section{Method}

Subjects. As in Experiment 1, the subjects were undergraduate students paid an hourly wage for their participation. Three of the subjects had participated in Experiment 1, and 5 had not.

Procedure. The procedures were similar to those used in Experiment 1 . The subjects judged which one of two locations, the index or the middle finger on the left hand, received a stimulus first. The same range of SOAs was tested.

In the first condition, static patterns were presented at both sites. The static pattern was generated by activating the middle two columns of the array from Row 2 to Row 15. This stimulus was equivalent to a single frame of the moving stimulus. It was presented for $65 \mathrm{msec}$. For these subjects, who were also tested in the second condition, we provided instructions and training to help improve their performances. The subjects were encouraged to discuss various cues that they found useful in judging temporal order. The experimenters described these and other cues that might be used in making TOJs. Trial-by-trial feedback was provided. The subjects were told about their overall performance on blocks of trials and how they were performing relative to other subjects. In a second condition, the subjects were tested with the same moving patterns as those used in the first two conditions of Experiment 1. As in the first condition, the subjects received instructions and feedback on their performances

In the third condition, the pair of stimuli consisted of a moving pattern, identical to the one used in Experiment 1, and a static pattern, identical to the static stimuli used in the first condition of Experiment 2. The moving stimulus could be presented so that the movement was either consistent or inconsistent with the temporal order of the patterns, and it could be presented as either the leading 
or the trailing stimulus. Thus, there were four types of trials: The moving stimulus could be presented first and be either consistent or inconsistent with the temporal order, or the moving stimulus could be presented second and be either consistent or inconsistent with the temporal order. The data were analyzed according to these four types of trials. Trial-by-trial feedback was provided in this condition. The subjects who participated in Condition 3 were also subjects in Conditions 1 and 2 and, thus, had received explicit training in TOJs.

Table 1 presents the number of subjects, blocks of trials, and the total number of trials per SOA for the three conditions tested in Experiment 2 .

\section{Results and Discussion}

The results from the first and second conditions, in which the subjects received explicit training in TOJs, are shown in Figure 6. The top panel shows the results with static patterns, and the bottom panel shows the results with moving patterns. As with the previous results, three functions are presented for the moving patterns: consistent, inconsistent, and overall. Training appears to have resulted in improved performance for both the static and the moving patterns. The threshold for the static patterns (75\% correct) was $45 \mathrm{msec}$, and that for the moving patterns was $48 \mathrm{msec}$. Performance in the static condition appears to have been similar to the mean performance level in the scanned condition, and, indeed, there is no significant difference between the two functions $[F(1,7)=$ $0.057, p=.82]$. Also, the similarity of the thresholds indicates that overall performance did not depend on whether the patterns were moving or static. The results also show that the bias was still evident even with improved overall performance and was still evident at SOAs of 52 and $100 \mathrm{msec}$, where performance was above $75 \%$ correct.

The results from the static/moving condition are shown in Figure 7. Panel A shows the results from those trials
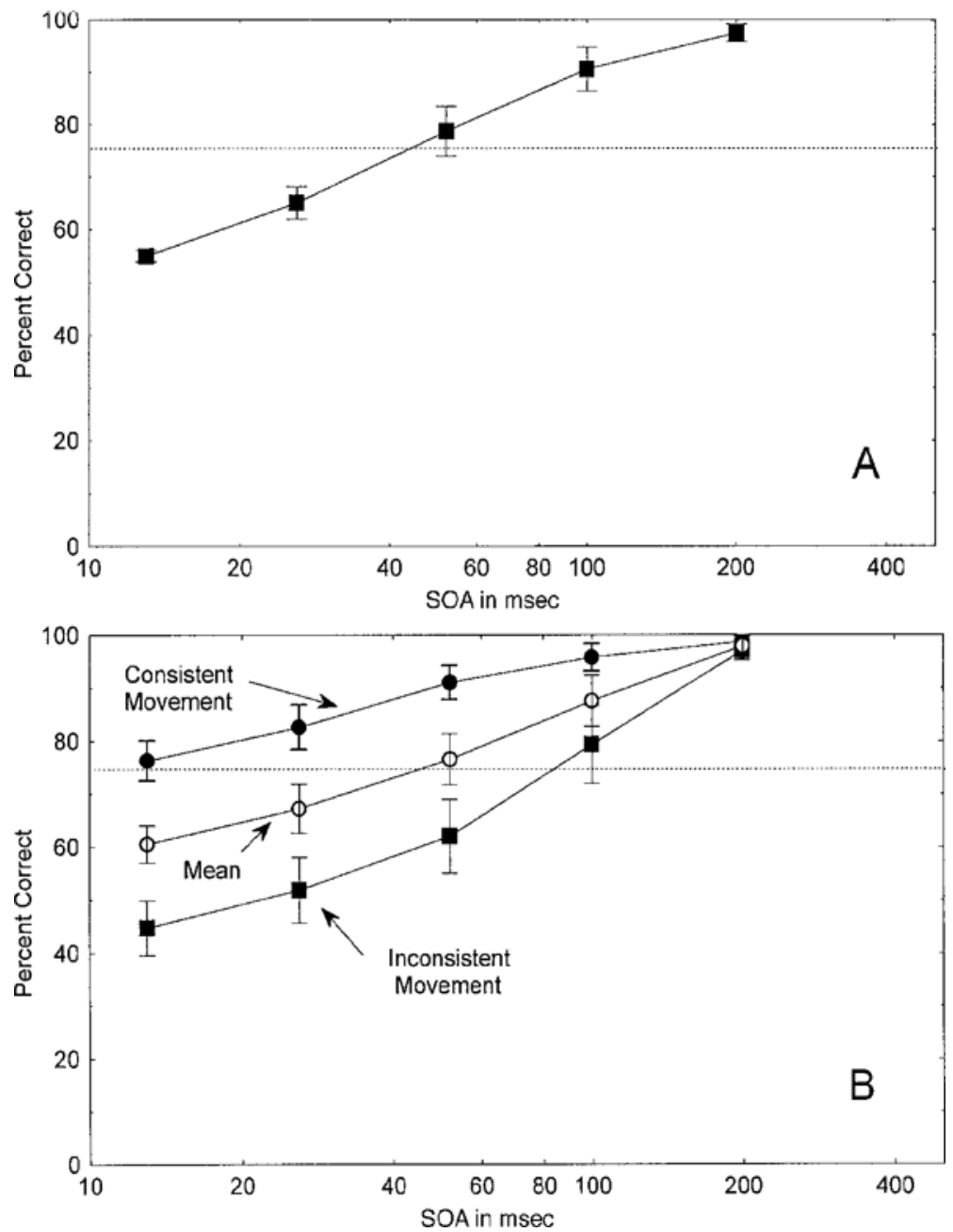

Figure 6. Performance in judging temporal order. Subjects received trial-by-trial feedback and training. The top panel represents results with static patterns. The bottom panel represents results with moving patterns. The top function represents consistent trials, the bottom function represents inconsistent trials, and the middle function represents the mean of the consistent and inconsistent trials. Error bars represent \pm 1 standard error of the mean. 

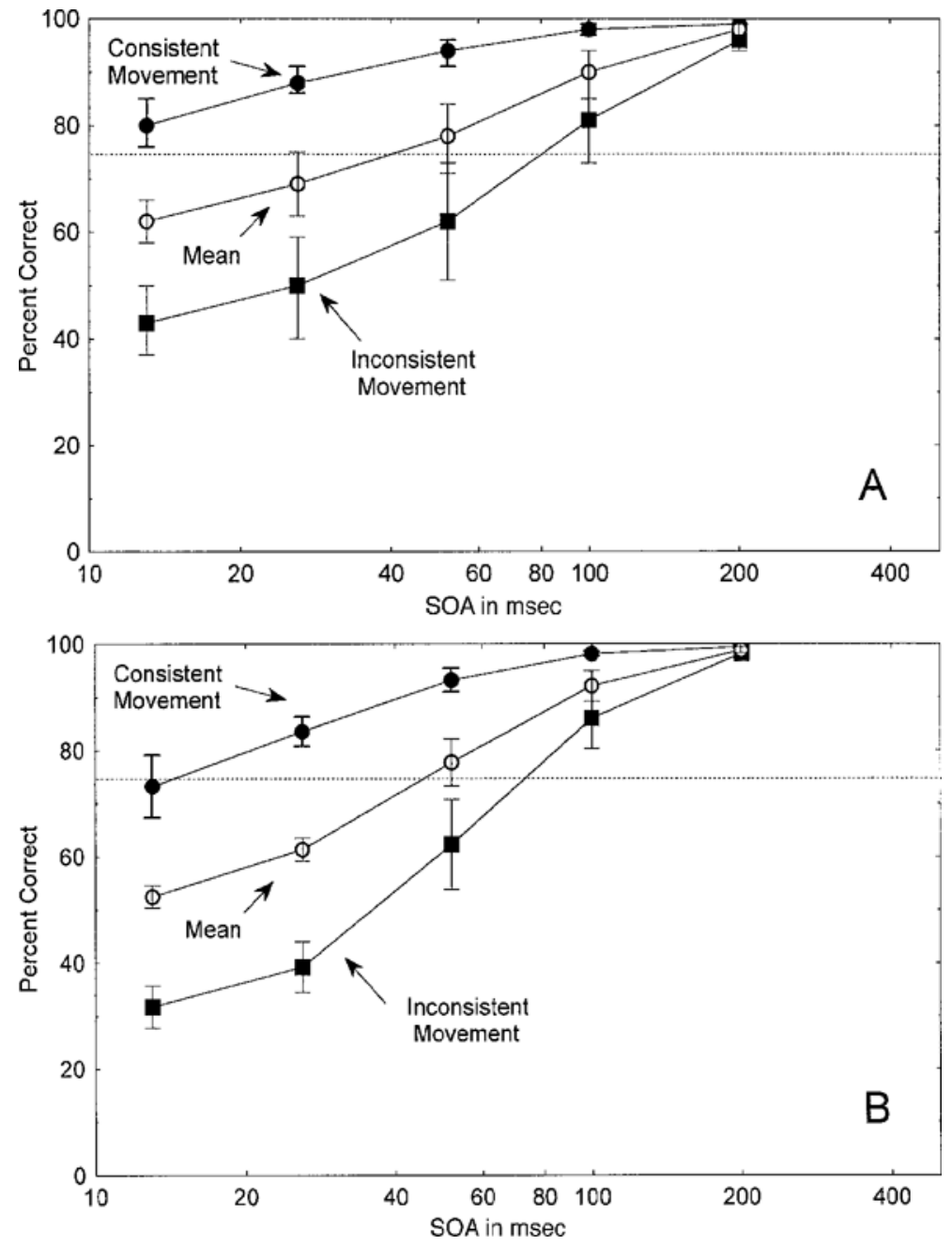

Figure 7. Performance in judging temporal order of a pair of patterns, one of which was moving and the other static. The top panel represents the trials on which the moving stimulus was presented first, followed by the static stimulus. The bottom panel represents the trials on which the static stimulus was presented first, followed by the moving stimulus. Error bars represent \pm 1 standard error of the mean.

on which the moving pattern was presented first. Panel B shows the results when the moving pattern was presented second. These measurements were designed to answer two questions: Was movement at both sites necessary to produce the bias in TOJs, and did a moving stimulus in the temporally leading position exert more of an influence on TOJs than did a moving stimulus in the trailing position? The answer to the first question is, clearly, no: Movement at a single site was sufficient to produce a large bias. Second, the bias is apparent whether the moving pattern was the leading or the trailing stimulus. One might have expected that the leading stimulus would exert a greater influence on the judgment than would the trailing stimulus. Such does not appear to have been the case. To get an estimate of the overall effect of pattern movement, we calculated the mean performance levels on the consistent function and the mean performance levels on the inconsistent function. The overall differences between the two functions were $26 \%$ when the moving pattern led and $27 \%$ when the static pattern led. An analysis of variance showed no main effect of the temporal position of the moving stimulus $[F(1,7)=1.1$, $p=.34]$. In addition, the $75 \%$ point was $43 \mathrm{msec}$ with the scanned pattern leading and $47 \mathrm{msec}$ with the scanned pattern trailing.

In Experiment 1, there was no bias when the movements at the two sites were in the opposite direction. When one of the two stimuli was a static pattern, there was still a considerable bias. These two sets of results indicate that when the movements at the two sites are in opposite directions (Experiment 1, Figure 5), the effects of the movements tend to cancel each other, resulting in no bias. 


\section{EXPERIMENT 3}

The results from Experiments 1 and 2 indicated that local movement substantially biases TOJs and that this bias is seen when the local movements at the two sites are in the same direction or when the movement at one location is not opposed by movement at a second location. The results are also consistent with the view that the bias is due to subjects' experiences with moving their hands over objects and the direction of hand motion (or relative object motion) being correlated with temporal order. In Experiment 3, the sites of stimulation were two fingers on opposite hands. With this bilateral condition, several questions were addressed. First, would local motion still affect TOJs when the sites are bilateral and the distance between sites is increased considerably? One hypothesis is that subjects are biased to base their TOJs on local movement because, in the course of haptic exploration, the temporal order and the direction of motion are almost always perfectly correlated. With bilateral haptic exploration, the correlation is much lower. The two hands often move in opposite directions when exploring an object. Second, with bilateral stimulation, the distance between the two sites of stimulation can be increased considerably. The bias might disappear because it depends on the close coupling of temporal and spatial cues that can operate only over a limited distance. In Experiment 3 , the displays generating the movement were placed at 28 and $80 \mathrm{~cm}$.

Bilateral stimulation also begins to address the neural basis for the interaction between two sites of stimulation. Essick and Whitsel (1992) recorded single-unit activity in SI to moving stimuli. Specifically, they examined the interaction between sites of stimulation within the same receptive field. The moving stimuli could be delivered so that both stimuli moved in the same direction or both in the opposite direction. The stimuli could be presented either simultaneously or sequentially. In some cases, the neural activity elicited by the two stimuli exceeded the vectorial sum of the activity elicited at a single site. In other cases, it fell below the vectorial sum. With ipsilateral stimulation of adjacent fingers, such as that used in Experiments 1 and 2, the sites may be within the same receptive fields of some neurons in SI. With bilateral stimulation, the likelihood that cells in SI will respond to stimulation at both sites is remote. If activity in SI, such as that described by Essick and Whitsel (1992), is necessary for the bias, then with bilateral stimulation, the bias should disappear.

\section{Method}

Subjects. As in Experiments 1 and 2, the subjects were undergraduate students who were paid an hourly wage. These subjects had not taken part in the training procedures in Experiment 2 that had resulted in improved performance.

Procedure. The procedure was similar to that used in Experiment 1. As in Experiment 1, there were two types of trials: those on which the moving patterns were consistent with the temporal order of the presentation of the patterns and those on which the movement was inconsistent with the order of presentation. In Experiment 3, the patterns were presented to the index fingers on the two hands. The displays were placed at two different distances. In the near condition, the displays were placed $28 \mathrm{~cm}$ apart, approximately shoulder width. In the far condition, the displays were placed $80 \mathrm{~cm}$ apart. In a third condition, the subjects were tested in the near condition and received trial-by-trial feedback. The subjects responded by means of foot pedals. The number of subjects tested, the number of sessions, and the total number of trials per SOA are shown in Table 1.

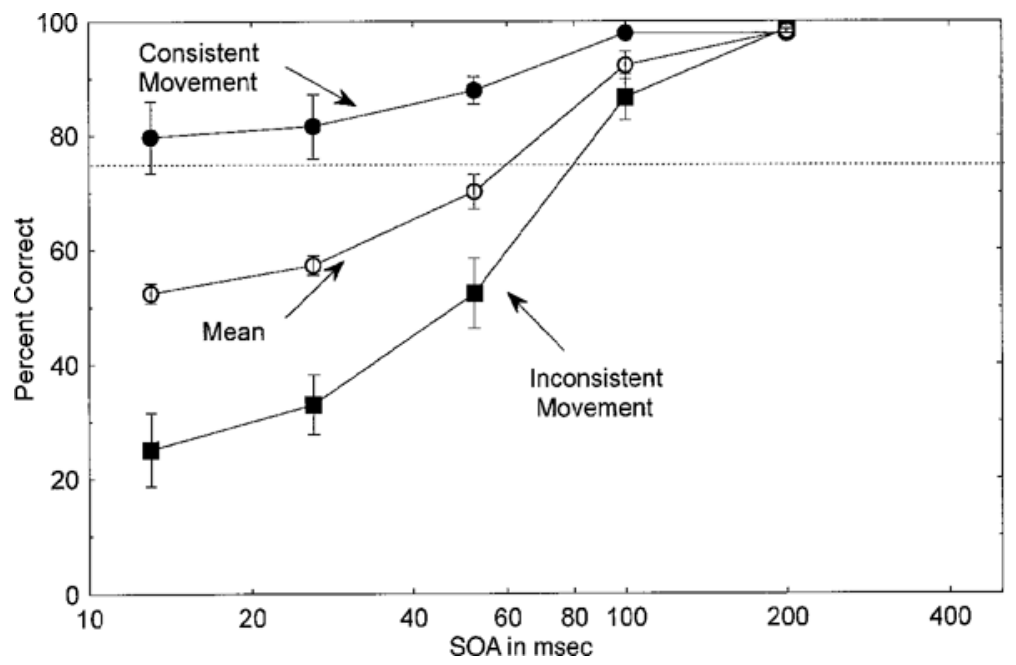

Figure 8. Performance in judging temporal order for stimuli presented bilaterally. The stimulus displays were $28 \mathrm{~cm}$ apart. The top function represents consistent movement, the bottom function inconsistent movement, and the middle function the mean of the consistent and inconsistent functions. The subjects did not receive trial-by-trial feedback. Error bars represent \pm 1 standard error of the mean. 


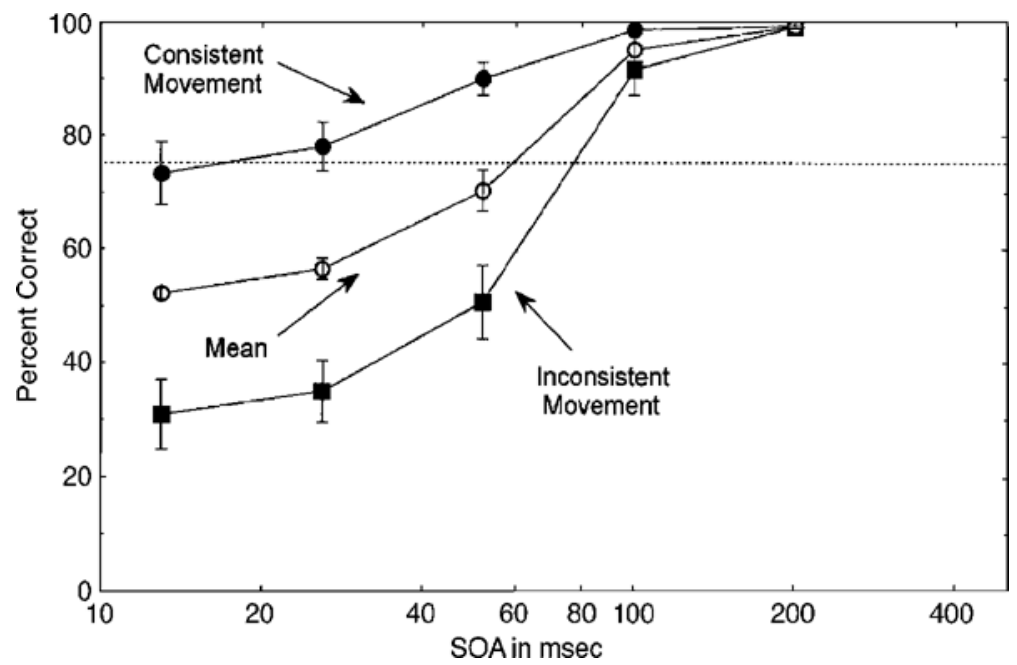

Figure 9. Performance in judging temporal order for stimuli presented bilaterally, with the addition of trial-by-trial feedback. The stimulus displays were $28 \mathrm{~cm}$ apart. The top function represents consistent movement, the bottom function inconsistent movement, and the middle function the mean of the consistent and inconsistent functions. Error bars represent \pm 1 standard error of the mean.

\section{Results and Discussion}

The results from the near condition with and without feedback and from the far condition are shown in Figures 8,9 , and 10 . The results are very similar to those seen in Experiment 1 with the ipsilateral stimulation. In these bilateral conditions, there continues to be a large bias favoring consistent movement [near condition without feedback, $F(1,5)=27.46, p<.01$; near condition with feedback, $F(1,7)=25.6, p<.01$; far condition, $F(1,5)=$ $6.75, p<.05]$. Also, there was no significant difference between the near condition without feedback (Figure 8) and the far condition [Figure $10 ; F(1,5)=0.13, p=.73$ ] Overall performance was quite similar for the three conditions. The only difference between these results and the other results with this paradigm is that 1 of the 6 subjects in the far condition showed a reverse bias-that is, this subject favored inconsistent, rather than consistent, directions of movement. The directions of movement were defined as they occurred in the frontal plane. If consistent and inconsistent had been defined as though the direc-

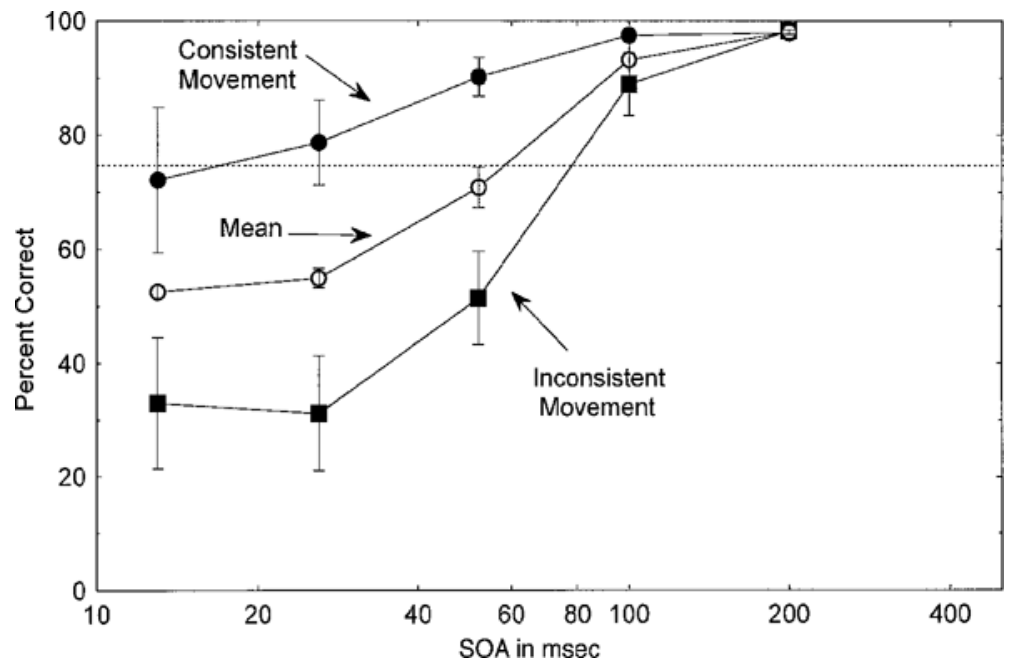

Figure 10. Performance in judging temporal order for stimuli presented bilaterally. The displays were $80 \mathrm{~cm}$ apart. The top function represents consistent movement, the bottom function inconsistent movement, and the middle function the mean of the consistent and inconsistent functions. Error bars represent \pm 1 standard error of the mean. 
tions of movement were passing behind the subject, the definitions of the two conditions would have been reversed. For this 1 subject, it is possible that the hands were separated sufficiently that the subject perceived the movement as though it were passing behind the body.

Taken together, the results of Experiment 3 are contrary to the view that the bias is closely related to haptic exploration. Only infrequently would haptic exploration of an object with two hands result in the same feature's passing beneath both index fingers with a brief temporal separation and with the hands moving in the same direction. Moreover, with the arms held widely apart, this type of haptic exploration is exceedingly rare. Also, for both bilateral conditions, but particularly for the far condition, the SOAs at which the same feature would pass beneath the two hands would increase substantially, as compared with the ipsilateral condition; yet there is no evidence that the temporal functions are altered in the bilateral data, as compared with the ipsilateral data.

The fact that shifting from ipsilateral to bilateral stimulation does not alter the TOJ bias suggests that the kind of neural activity recorded by Essick and Whitsel (1992) in SI is not necessary for the bias. Interactions between bilateral sites suggest that later stages in processing are involved and are consistent with a response bias explanation.

\section{EXPERIMENT 4}

In Experiment 4, we asked whether the effect of movement on TOJs is limited to the tactile modality. Specifically, would similar effects be seen with visual stimuli? With both tactile and visual stimuli, spatial information is registered directly on the sensory surface; and, as with tactile stimuli, temporal order and movement are highly correlated. As an object moves across the visual field, the direction of motion nearly always predicts the temporal order.

\section{Method}

Subjects. The subjects were undergraduate students at Indiana University. Nine of the subjects had previous experience in judging tactile temporal order. Three of the subjects had no previous experience in making TOJs.

Apparatus. The visual stimuli were generated on a point-plotting oscilloscope with a 1-msec refresh rate and an extremely rapid phosphor decay (P15 phosphor). The subjects were seated $70 \mathrm{~cm}$ away from the screen in a dimly lit room. Viewing was free, and all the subjects had normal or corrected-to-normal vision, with no history of other visual disorders. The point-plotting oscilloscope can present single dots at any one of 4,096 positions on the horizontal and vertical dimensions. A schematic diagram of the display appears in Figure 11 and shows the four always-present fixation points and the two patterns (lines). The luminance of the background was $1.4 \mathrm{~cd} / \mathrm{m}^{2}$ and the luminance of a single dot was $104 \mathrm{~cd} / \mathrm{m}^{2}$.

Procedure. The subject's task was to indicate whether the top or the bottom pattern was presented first. The patterns were generated by sequentially stimulating the dots in between the start and endpoints of each pattern. At any given millisecond, only a single dot was presented in each pattern (if there were any illumination of the screen at all). The stimulated dot was sequentially moved from the starting to the ending position over a period of $65 \mathrm{msec}$, which gives the appearance of movement within each pattern. The top and bottom patterns appeared as two lines, quickly drawn on the screen. Following the presentation of both patterns, the subject indicated whether the top pattern appeared first (by pressing a " 1 " on the keypad) or the bottom pattern appeared first (by pressing a " 2 " on the keypad). A second keypress initiated the next trial. No feedback was provided.

The motion of the dots could be either consistent with the temporal order (and global motion) of the two patterns or inconsistent. In the consistent condition, for a top-first trial, the dots within the patterns moved in a downward direction. In a bottom-first trial, the dots within a pattern moved in an upward direction. The inconsistent condition reversed the direction of motions, so that a top-first trial would have motions within the pattern going up and a bottom-

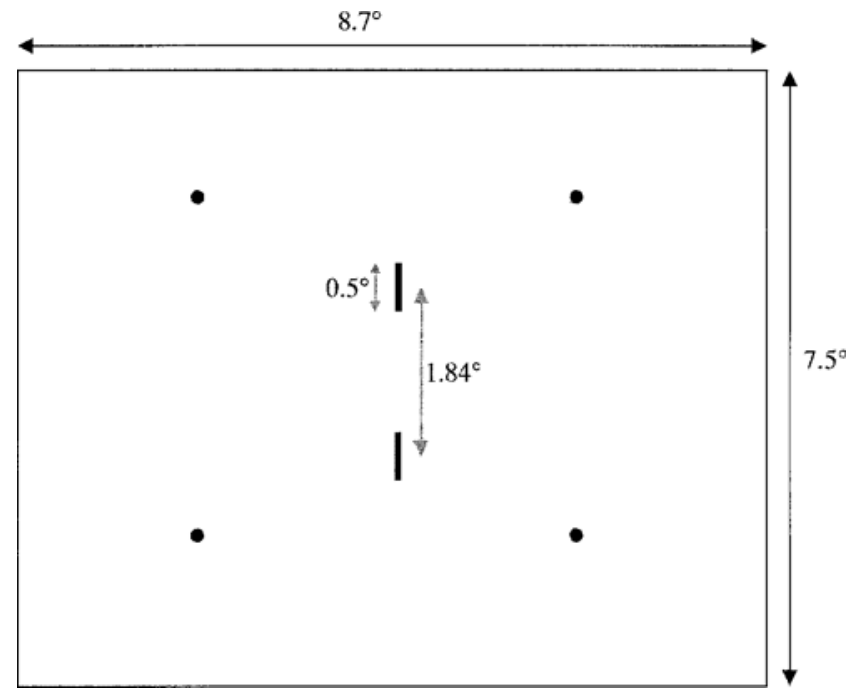

Figure 11. A schematic diagram of the visual display. 
first trial would have them going down. The four types of trials (consistent and inconsistent crossed with top first and bottom first) were presented an equal number of times in pseudorandom order.

The SOA between the first and the second patterns was set to a single value within a block of trials. The subjects began with a block of 32 trials at a $400-\mathrm{msec}$ SOA, followed by blocks with SOAs of $200,100,50,25$, and $13 \mathrm{msec}$. This process was repeated five times per subject, usually conducted in two 30 -min sessions. The subjects began with the longest SOA, which reinforced the instructions that direction of motion within a pattern was irrelevant to the TOJ. The subjects completed 80 trials per condition per SOA.

\section{Results and Discussion}

Of the 12 subjects tested, 9 had participated in some of the previous tactile experiments, and 3 had not. The performances from the two groups of subjects, 9 experienced and 3 naive, were similar. The results shown in Figure 12 represent the mean performance from all 12 subjects. The data are plotted as in Figure 3. All the subjects showed considerably better performance on the consistent trials than on the inconsistent trials. There were significant effects of trial type $[F(1,11)=30.40$, $p<.001]$ and SOA $[F(5,55)=196.23, p<.001]$ and a significant interaction $[F(5,55)=21.5, p<.001]$. The aim of Experiment 4 was to see whether movement would affect TOJs for visual stimuli in the same way that it affected TOJs for tactile stimuli. The similarity of the visual results to the tactile results suggests that similar processes may affect both modalities.

\section{GENERAL DISCUSSION}

The present findings, which demonstrate that variations in an irrelevant dimension, movement, can affect perfor- mance in processing another dimension, share commonalities with studies that address both Garner interference/ facilitation (1974) and Stroop-like tasks. Garner interference occurs when variability is introduced on an irrelevant dimension and performance is reduced relative to a baseline condition with fixed values on the irrelevant dimension. We find both congruence and interference effects depending on the value of the irrelevant dimension. In a Stroop-like task, Clark and Brownell (1976) had subjects indicate whether an arrow was pointing up or down. The arrow was placed at various heights within a rectangle. The subjects were faster in judging an upward-pointing arrow the higher the arrow was in the rectangle. Similarly, downward-pointing arrows were judged faster the lower they were in the rectangle. The present findings are also similar to results obtained in cross-modal congruence and interference studies. In such studies, subjects have classified, for example, the spatial position of a visual stimulus. The visual stimulus was accompanied by an irrelevant auditory stimulus. High-pitched tones speeded classification of high-positioned visual stimuli and slowed classification of low-positioned visual stimuli (Ben-Artzi \& Marks, 1995). In the present study, accuracy, rather than speed of classification, was measured, making direct comparisons with previous studies problematic; however, one might imagine that the subjects in the present study would have been able to judge the direction of motion on the skin more rapidly if the temporal order of the two sites had been consistent with that motion.

The results are also consistent with previous studies of the perception of temporal order in showing how labile TOJs can be. Yamamoto and Kitazawa (2001a, 2001b) examined the effect that the position of the hands had on

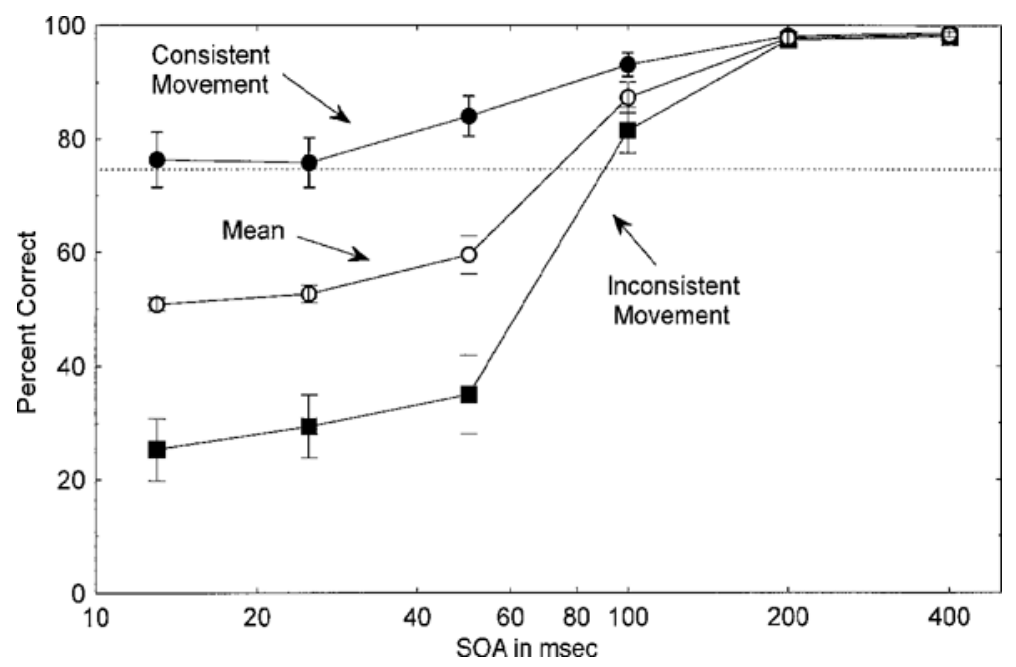

Figure 12. Performance in judging the temporal order of visual stimuli. The top function represents trials on which the direction of motion of the patterns was consistent with the order of presentation. The bottom function represents trials on which the direction of motion of the patterns was inconsistent with the order of presentation. The middle function represents the mean of the consistent and inconsistent functions. Error bars represent \pm 1 standard error of the mean. 
TOJs. In these studies, subjects had to judge which one of two hands received a tactile stimulus first. Crossing the hands produced substantial declines in sensitivity in TOJs. Subjects erred in judging temporal order even when the two stimuli were separated by more than $300 \mathrm{msec}$. Subjects did not receive trial-by-trial feedback in these studies. A significant difference between the present study and studies that demonstrate the lability of TOJs is that even though inconsistent movement produces incorrect responses, the overall sensitivity remains unchanged. Subjects are equally sensitive whether static or moving patterns are used (Figure 6). Because movement does not reduce sensitivity and because movement and temporal order are highly correlated for both haptic and visual stimuli, using the direction of movement will generally lead to correct judgments of temporal order, at least for haptic exploration with a single hand. Moreover, the main effect of movement is seen at the briefer SOAs, where subjects' performances approach chance levels. At brief SOAs, where subjects will be forced to guess, relying on direction of movement in haptic exploration or visual judgments will improve performance. A braille reader, for example, might misread a word because the order of two letters is reversed as they move across two fingers. The close coupling of the direction of movement and TOJs would prevent people from judging incorrectly which finger received the stimulus first.

Also consistent with other studies of temporal order, the subjects in the present study often reported that they relied on apparent motion in judging temporal order and, furthermore, that the moving stimuli seemed to interfere with the apparent motion cue. Essick and Whitsel (1988) examined the ability of subjects to discriminate the direction of motion at one location on the hand when a moving stimulus was presented to a second location. One of the findings was that when the two stimuli were delivered with a temporal separation between them, performance was interfered with. The interference was due, in part, to apparent motion being generated between the two sites, what the authors refer to as "long-range cues." In other words, the long-range cues interfered with judgments of the direction of motion at the test site. The present results suggest a reverse effect: Local patterns of motion can affect the perception of apparent motion and, in that way, either enhance or interfere with TOJs. The suggestion is that when the movement at the fingerpads is consistent with the apparent motion between the two sites, the perception of the direction of apparent motion is enhanced, and subjects respond accordingly and correctly. Inconsistent movement interferes with or may, in fact, create the percept of apparent motion in the opposite direction, with subjects responding accordingly and incorrectly. The fact that apparent motion can be obtained with bilateral stimulation (Sherrick, 1968a) is in agreement with the present findings that TOJs can be affected by moving stimuli applied either ipsilaterally or bilaterally. It should be noted that bilateral apparent motion is not as compelling as motion generated ipsilaterally (Sherrick, 1968a).
To the extent that apparent motion is playing a role in the biasing of TOJs, it might be affecting TOJs in the following way. In making TOJs with static stimuli, subjects report that the brief temporal separation between patterns causes their attention to be drawn from one location to another. Subjects also report using this direction of movement in making their TOJs, the attentional shift being from the first to the second stimulus. With moving patterns, the direction of motion at the local site may produce a shift in attention, exogenous cuing, that is either consistent or inconsistent with the temporal order of the patterns. This shift in attention may produce a corresponding change in performance. Some observations with visual stimuli support the view that moving stimuli may affect attentional shifts. It has been observed that a moving visual stimulus is perceived at a location ahead of its actual location (Baldo \& Klein, 1995; Purushothaman, Patel, Bedell, \& Ogmen, 1998). This has been described as though the observer is anticipating the trajectory of the moving stimulus and perceiving it where it will be, rather than where it is. The extent to which this effect is due to higher level processes such as attention is, however, unclear (Khurana \& Nijhawan, 1995; Whitney \& Murakami, 1998). There is evidence that the neural basis for this effect may begin in the retina (Berry, Brivanlou, Jordan, \& Meister, 1999). In the present experiments, the subjects were never given instructions about attending to one location or the other or about attending to the local patterns of motion in any particular way. The fact that all the subjects showed reliable effects suggests that any attentional effects are tied closely to the stimulus conditions and operate at fairly low levels.

Although likely to be operating at relatively low levels, the tactile temporal order effects may be used to address higher level issues, such as how the position of the limbs in space affects the definition of consistent and inconsistent motion. With the tactile arrays placed directly in front of the subjects, as they were in the present experiments, the definition of consistent and inconsistent motion was fairly straightforward; however, the position of the hands and displays can be altered spatially. For example, one can rotate the displays so that rather than being parallel to each other, they are placed at angles of $30^{\circ}, 60^{\circ}$, or $90^{\circ}$. Are the biases in the TOJs altered as the angle between the two hands (and displays) is changed? Does the perception of the direction of motion (consistent/inconsistent) change as limb position changes? TOJs may be used as a way to explore the effect of kinesthetic information on the perception of tactile motion.

\section{REFERENCES}

BALdo, M. V., \& KLEIN, S. A. (1995). Extrapolation or attention shift? Nature, 378, 565-566.

Ben-Artzi, E., \& Marks, L. E. (1995). Visual-auditory interaction in speeded classification: Role of stimulus difference. Perception \& Psychophysics, 57, 1151-1162.

Berry, M. J., II, Brivanlou, I. H., Jordan, T. A., \& Meister, M. (1999). Anticipation of moving stimuli by the retina. Nature, 398, 334-338.

Bliss, J. C., Katcher, M. H., Rogers, C. H., \& Shepard, R. P. (1970). 
Optical-to-tactile image conversion for the blind. IEEE Transactions on Man-Machine Systems, MMS-11, 58-64.

Clark, H. H., \& Brownell, H. H. (1976). Position, direction, and their perceptual integrality. Perception \& Psychophysics, 19, 328-334.

Craig, J. C. (1980). Modes of vibrotactile pattern perception. Journal of Experimental Psychology: Human Perception \& Performance, 6 , 151-166.

Craig, J. C. (2002). Identification of scanned and static patterns. Perception \& Psychophysics, 64, 107-120.

Craig, J. C., \& XU, B. (1990). Temporal order and tactile patterns. Perception \& Psychophysics, 47, 22-34.

Essick, G. K., \& WHITSEL, B. L. (1988). The capacity of human subjects to process directional information provided at two skin sites. Somatosensory \& Motor Research, 6, 1-20.

Essick, G. K., \& WhitseL, B. L. (1992). The response of SI directionally selective neurons to stimulus motion occurring at two sites within the receptive field. Somatosensory \& Motor Research, 10, 97-113.

Gardner, E. P., \& SkLAR, B. F. (1994). Discrimination of the direction of motion on the human hand: A psychophysical study of stimulation parameters. Journal of Neurophysiology, 71, 2414-2429.

GARNER, W. R. (1974). The processing of information and structure. Potomac, MD: Erlbaum.

HiLl, J. W. (1971). Processing of tactual and visual point stimuli sequentially presented at high rates. Journal of Experimental Psychology, 88, 340-348.

HiLl, J. W., \& BLISs, J. C. (1968). Perception of sequentially presented tactile point stimuli. Perception \& Psychophysics, 4, 289-295.
Hirsh, I. J., \& Sherrick, C. E. (1961). Perceived order in different sense modalities. Journal of Experimental Psychology, 62, 423-432.

Khurana, B., \& NiJHAWAN, R. (1995). Extrapolation or attention shift? Nature, 378, 566.

Marks, L. E., Girvin, J. P., O'Keefe, M. D., Ning, P., Quest, D. O., Atunes, J. L., \& Dobelle, W. H. (1982). Electrocutaneous stimulation: III. The perception of temporal order. Perception \& Psychophysics, 32, 537-541.

Purushothaman, G., Patel, S. S., Bedell, H. F., \& Ogmen, H. (1998). Moving ahead through differential visual latency. Nature, 396, 424.

Sherrick, C. E. (1968a). Bilateral apparent haptic movement. Perception \& Psychophysics, 4, 159-160.

SHerrick, C. E. (1968b). Studies of apparent tactual movement. In D. R. Kenshalo (Ed.), The skin senses (pp. 331-344). Springfield, IL: Thomas.

Sherrick, C. E. (1970). Temporal ordering of events in haptic space. IEEE Transactions on Man-Machine Systems, MMS-11, 25-28.

Whitney, D., \& Murakami, I. (1998). Latency difference, not spatial extrapolation. Nature Neuroscience, 1, 656-657.

Yamamoto, S., \& KitaZawa, S. (2001a). Reversal of subjective temporal order due to arm crossing. Nature Neuroscience, 4, 759-765.

Yamamoto, S., \& KitaZawa, S. (2001b). Sensations at the tips of invisible tools. Nature Neuroscience, 4, 979-980.

(Manuscript received December 13, 2001; revision accepted for publication June 12, 2002.) 